\title{
Modelling of undrained shearing of soft natural clays
}

\author{
Alexandros L. Petalas ${ }^{1, *}$, Mats Karlsson ${ }^{1}$, and Minna Karstunen ${ }^{1}$ \\ ${ }^{1}$ Chalmers University of Technology, Dept. of Architecture and Civil Engineering, Sweden
}

\begin{abstract}
. stress-strain response of soft natural clays is characterised by anisotropy, destructuration and rate-dependency. An accurate constitutive description of these materials should take into consideration all of the characteristics above. In this paper, two constitutive models for soft soils, namely the SCLAY1S and Creep-SCLAY1S models are used to simulate the undrained response of two soft natural clays, Gothenburg clay from Sweden and Otaniemi clay from Finland. The SCLAY1S model accounts for the effect of inherent and induced anisotropy and destructuration, while the Creep-SCLAY1S accounts also for the creep and rate effects. The model simulations are compared against triaxial compression and extension tests on anisotropically consolidated samples. The results demonstrate the need to incorporate all features represented in the Creep-SCLAY1S model when modelling structured natural clays.
\end{abstract}

\section{Introduction}

The mechanical response of soft natural clays is affected by their micro-structure that is often anisotropic, and the apparent inter-particle bonding. Moreover, they exhibit rate-dependent behaviour. The initial anisotropy of marine clays is often developed under $K_{0}$-conditions (initial deposition, self-weight consolidation and aging), and the anisotropy evolves during subsequent loading, if the loading path differs from the $K_{0}$-condition. In addition to the evolving anisotropy, when irrecoverable straining occurs and the particle cluster and contacts evolve, the interparticle bonding degrades. This process is called destructuration [1].

Two constitutive models, namely SCLAYS-1S [2] and Creep-SCLAY1S [3, 4] are used in this paper for simulating triaxial loading paths on two soft natural clays (Gothenburg and Otaniemi clays [5]). The elasto-plastic SCLAY1S model accounts for inherent and evolving anisotropy by utilising a rotational hardening law, proposed in [6]. In addition to anisotropy, the model accounts for bonding and destructuration by using the concept of an intrinsic yield surface [7]. Finally, the rate-dependent elasto-visco-plastic Creep-SCLAY1S model accounts not only for anisotropy, bonding and destructuration, but also for rate-effects.

The paper is organised as follows: In Section 2 the two models are briefly presented, and the model parameters for the numerical simulations are discussed. In Section 3, data from triaxial compression and extension tests on anisotropically consolidated Gothenburg clay and their numerical simulations are presented. The effects of destructuration, anisotropy and shearing rate are highlighted. The results demonstrate the need to incorporate all features represented in the Creep-SCLAY1S model. Consequently,

*e-mail: petalas@chalmers.se in Section 4, three anisotropically consolidated undrained triaxial tests on Otaniemi clay are simulated, in which samples from the same depth have been exposed to different levels of anisotropic consolidation, before shearing to failure. These simulations highlight the need to account for the stress history and the degradation of bonding in the subsequent simulations.

\section{Constitutive models and calibration}

The SCLAY1S model [2] and the Creep-SCLAY1S [3, 4] model are presented schematically in triaxial space (Fig. 1) for the special case of a cross-anisotropic material with the main axis of the fabric coinciding with the principal stress directions in the triaxial test. The yield surface of SCLAY1S is a sheared ellipse [8], with the size defined by $p_{m}^{\prime}$ and the inclination by $\alpha$, a scalar measure of anisotropy for the special case of triaxial conditions. A fabric tensor is used in the generalised formulation, (see [6]). The imaginary intrinsic yield surface represents the yield surface of the same soil, with the same void ratio and stress history, but without the bonds. The size of the intrinsic yield surface is controlled by the state variable $p_{m i}^{\prime}$, with the same inclination as the the yield surface of the natural clay. The sizes of the two surfaces are related via state parameter $\chi$ by $p_{m}^{\prime}=\chi p_{m i}^{\prime}$.

Both the anisotropy, represented by $\alpha$, and the amount of bonding $\chi$ evolve due to irrecoverable straining. $\alpha$ evolves towards a target value based on the current state of the soil, and hence the yield surface rotates, controlled by a rotational hardening law (see [6] for details). $\chi$ evolves from the initial value $\chi_{0}$ towards zero due to destructuration. The elastic response of the model is assumed to be non-linear with a stress dependent elastic formulation, similar to the Modified Cam Clay model.

In the Creep-SCLAY1S model (Fig. 1), the yield surface is replaced by the Normal Compression Surface 
(NCS), with the size defined by $p_{p}^{\prime}$. An additional surface, representing the current (effective) stress state (CSS) is used, with the size defined by $p_{e q}^{\prime}$, and the same inclination $\alpha$ as the NCS. The current stress state is always on the CSS. The distance of $p_{e q}^{\prime}$ with respect to $p_{p}^{\prime}$ relates to the magnitude of visco-plastic strains that are computed via an explicitly defined visco-plastic multiplier [9]. CreepSCLAY1S does not predict purely elastic response, however, for $p_{e q}^{\prime}<p_{p}^{\prime}$, the magnitude of the predicted viscoplastic strain is very small. The rotational hardening and destructuration laws are the same as in the SCLAY1S model.

The model parameters are presented in Table 1. The calibration of the models are explained in detail in [2$4,6,10]$. In this work, in order to investigate the effect of destructuration, anisotropy and shearing rate, the complexity of the models is hierarchically increased. When $\alpha_{0}=0, \omega=0$ and $\chi_{0}=0$ and the compressibility of the natural clay $\lambda$ is used instead of the intrinsic value $\lambda_{i}$, the SCLAY1S model becomes equivalent to a Lode angle -dependent version of the Modified Cam Clay model (called MCC in the legends of the figures). When $\chi_{0}>0$ to account for the effect of bonding, the model is an isotropic model with destructuration (called Structure in the legends). By using $\chi_{0}>0, \alpha_{0}>0$ and $\omega>0$ the effect of both destructuration and anisotropy are taken into account (called Anisotropy in the legends). Finally, all the above and the rate effects are taken into account via the CreepSCLAY1S model (called Creep in the legends), which instead of $\lambda_{i}$ uses the modified intrinsic compression index $\lambda^{\star}$, and the volumetric compression in the purely elastic range is represented by the modified swelling index $\kappa^{\star}$.

\section{Undrained response of Gothenburg clay}

In Figs. $2 \mathrm{a}$ and $2 \mathrm{~b}$ the simulations of a triaxial compression and a triaxial extension test, respectively, are presented. The effective stress and the void ratio of the soil after the anisotropic consolidation and the rate of undrained shearing are summarised in Table 2. It can be concluded that in triaxial shearing, the destrcucturation law plays a significant role. The equivalent MCC model cannot simulate the undrained softening response after the peak $q$. When anisotropy is taken into account via the rotational hardening law (Anisotropy and Creep legends) the results become even more accurate, as far as the undrained triaxial extension response is concerned. The MCC model and the MCC with structure over-predict the purely elastic response as can be seen in Fig. 2bb. Finally, the CreepSCLAY1S (Solid grey line) predicts a slightly higher peak due to the strain- rate effect, but reproduces very accurately the anisotropic response and destructuration. This is suggesting that the assumed value for $M_{c}$ is too high for the creep model, perhaps representing the peak rather than the critical state value.
Table 1. Value of the model parameters for Gothenburg and Otaniemi clays.

\begin{tabular}{|c|c|c|c|}
\hline & \multicolumn{2}{|c|}{ Gothenburg Clay } & Otaniemi clay \\
\hline Parameter & SCLAY-1S & $\begin{array}{c}\text { Creep } \\
\text { SCLAY-1S }\end{array}$ & $\begin{array}{c}\text { Creep } \\
\text { SCLAY-1S }\end{array}$ \\
\hline$\lambda[-]$ & 0.6 & $\lambda^{\star}=0.29$ & $\lambda^{\star}=0.26$ \\
$\lambda_{i}[-]$ & 0.24 & $\lambda_{i}^{\star}=0.08$ & $\lambda_{i}^{\star}=0.063$ \\
$\kappa[-]$ & 0.03 & $\kappa^{\star}=0.01$ & $\kappa^{\star}=0.012$ \\
$\nu[-]$ & 0.2 & 0.2 & 0.25 \\
$M_{c}[-]$ & 1.4 & 1.4 & 1.3 \\
$M_{e}[-]$ & 1.1 & 1.1 & - \\
$\omega[-]$ & 300 & 300 & 20 \\
$\omega_{d}[-]$ & 0.95 & 0.95 & 0.86 \\
$\xi[-]$ & 8 & 8 & 15 \\
$\xi_{d}[-]$ & 0.5 & 0.5 & 0.2 \\
$\alpha_{0}[-]$ & 0.54 & 0.54 & 0.5 \\
$\chi_{0}[-]$ & 8 & 8 & 8 \\
$\tau[$ days $]$ & 1 & 1 & 1 \\
$\mu[-]$ & 0.00125 & 0.00125 & 0.00125 \\
\hline
\end{tabular}

Table 2. Assumed initial conditions for triaxial compression and extension tests on Gothenburg clay. $i$ : after consolidation, before shearing.

\begin{tabular}{|c|c|c|c|c|c|c|}
\hline Test & $e_{i}$ & $\begin{array}{c}\sigma_{a, i}^{\prime} \\
{[\mathrm{kPa}]}\end{array}$ & $\begin{array}{c}\sigma_{r, i}^{\prime} \\
{[\mathrm{kPa}]}\end{array}$ & $K_{0}$ & OCR & $\begin{array}{c}\text { Shear rate } \\
{[\mathrm{mm} / \mathrm{min}]}\end{array}$ \\
\hline $\mathrm{TC}$ & 1.87 & 167 & 101.65 & 0.61 & 1.25 & 0.01 \\
$\mathrm{TE}$ & 1.95 & 176.61 & 101.65 & 0.58 & 1.25 & 0.01 \\
\hline
\end{tabular}

\section{Undrained response of Otaniemi clay}

Three undrained triaxial shear tests on natural Otaniemi clay are simulated with the use of the PLAXIS finite element software and the Creep-SCLAY1S model as boundary value problems. The clay samples are extracted from the same depth and the Normal Compression Surface (NCS) is initialised by using the in-situ vertical effective stress $\left(\sigma_{v}^{\prime}=25\right)$ and $K_{0 N C}$ calculated via the Jaky's formula. The initial void ratio $e_{0}$ (before the anisotropic consolidation), the void ratio at the start of shearing $e_{i}$ and the effective stress state after the anisotropic consolidation, as well as the rate of shearing are summarised in Table 3.

The numerical simulations are performed in two stages. At the first stage the anisotropic consolidation is simulated. For the samples Ota2239 and Ota2240 the stress state after anisotropic consolidation is outside of the NCS, initialised by the apparent preconsolidation pressure of the natural clay, and thus, the NCS evolves during anisotropic consolidation. As the stress ratio used in the 

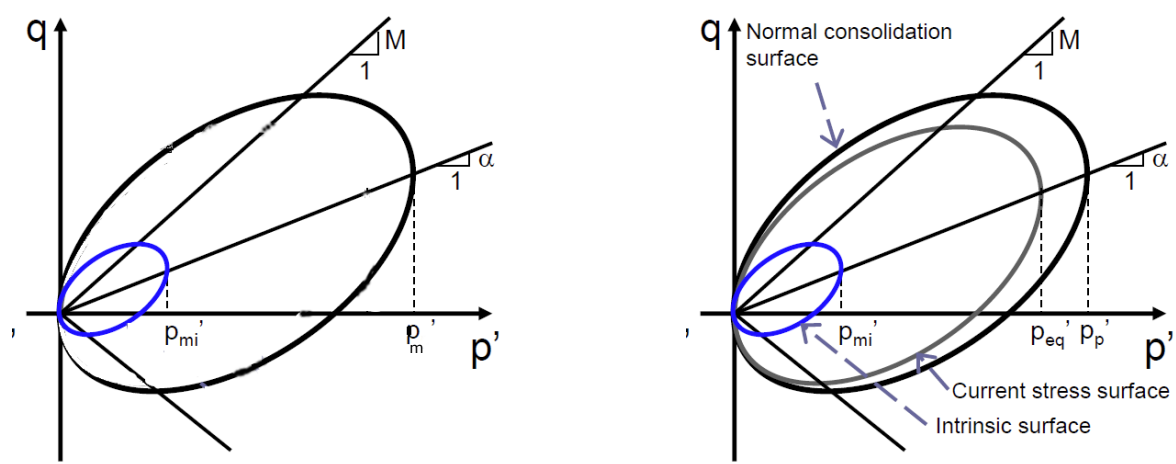

Fig. 1. Constitutive models. Left: SCLAY-1S; right: Creep-SCLAY1S.

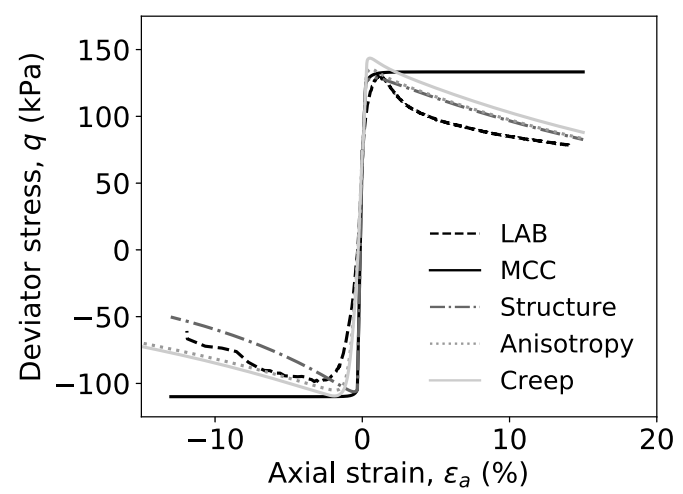

(a) Stress-strain curves

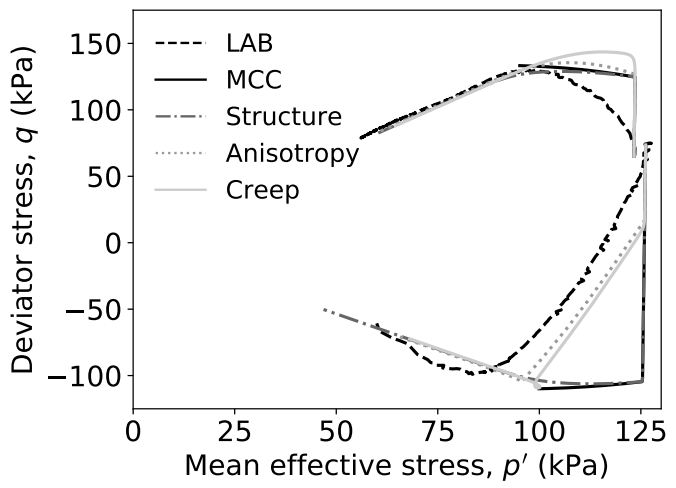

(b) Stress-path

Fig. 2. Undrained triaxial shearing of Gothenbug clay and numerical simulations with evolving model complexity.

anisotropic consolidation differs from the $K_{0}$ consolidation, NCS is also expected to rotate during consolidation. At the second stage, undrained shearing is applied. The results of the simulations are presented in Figs. 3 and 3. There is good agreement of the results from the numerical analysis of Ota2238 test but the model overestimates the undrained strength for tests Ota2239 and Ota2240. Three additional dashed lines are presented in both Fig. 3 and 3 for additional simulations that do not take into account the anisotropic consolidation stage. In those simulations the inclination of the NCS estimated by the in-situ conditions is used ( $\alpha=0.5$ ) without taking into account any evolution during anisotropic consolidation. Moreover, the initial value of the destructuration parameter $\chi_{0}=8$ and it's evolution is also not taken into account. It can be concluded from Fig. 3 and 3 that not including the evolution of the state variables during anisotropic consolidation significantly decreases the accuracy of the predictions. The numerical simulations can be improved if more data for the initial inclination of the NCS and it's evolution are available.

Fig. 4a demonstrates how the model predicts the anisotropy $\alpha$ decreases significantly during consolidation for Ota2289 and Ota2240, due to the high applied value of $K_{0} \approx 0.8$, and consequently NCS is predicted to rotates towards the isotropic state. During the undrained shearing anisotropy evolves again, with an increasing $\alpha$-value predicted. The bonding parameter $\chi$ (Fig. $4 \mathrm{~b}$ ) is also predicted to decreases significantly both during the anisotropic consolidation and the undrained shearing. Consequently, it is very important to simulate both stages in these triaxial tests as the initial consolidation will also change the initial state if the clay is allowed to yield during the consolidation.

Table 3. Initial conditions for triaxial compression of Otaniemi clay. 0: before consolidation; $i$ after consolidation, before shearing.

\begin{tabular}{|c|c|c|c|c|c|c|}
\hline Test & $e_{0}$ & $e_{i}$ & $\begin{array}{c}q_{i} \\
{[\mathrm{kPa}]}\end{array}$ & $\begin{array}{c}p_{i}^{\prime} \\
{[\mathrm{kPa}]}\end{array}$ & $K_{0}$ & $\begin{array}{c}\text { Shear rate } \\
{[\% / \mathrm{h}]}\end{array}$ \\
\hline Ota2238 & 3.28 & 3.14 & 3 & 15.9 & 0.83 & 0.63 \\
Ota2239 & 3.47 & 2.76 & 6 & 27.7 & 0.82 & 0.64 \\
Ota2240 & 3.32 & 3.32 & 9 & 41.7 & 0.81 & 0.061 \\
\hline
\end{tabular}




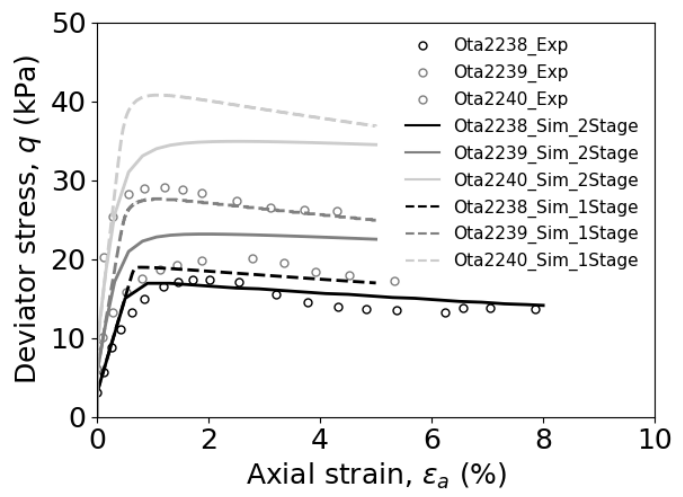

(a) Stress-strain curves

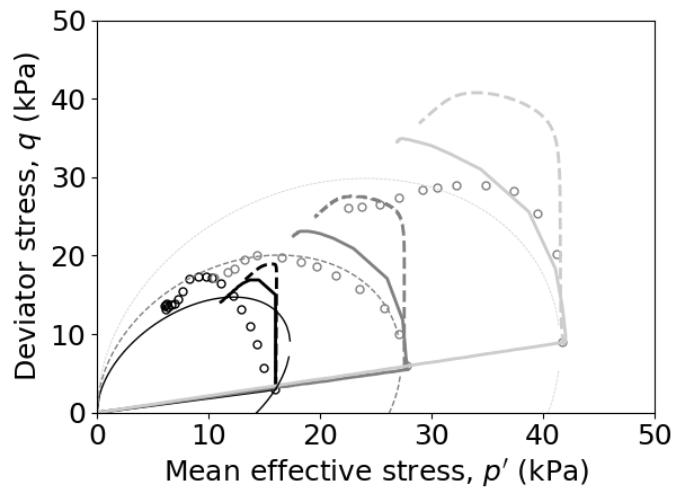

(b) Stress-path

Fig. 3. Undrained shearing tests on Otaniemi clay and numerical simulations with Creep-SCLAY1S. Solid lines: Simulating both the anisotropic consolidation and the undrained shearing stages (2 stages). Dotted lines: Simulating only the undrained shearing stage (1 stage). Lab data after [5].

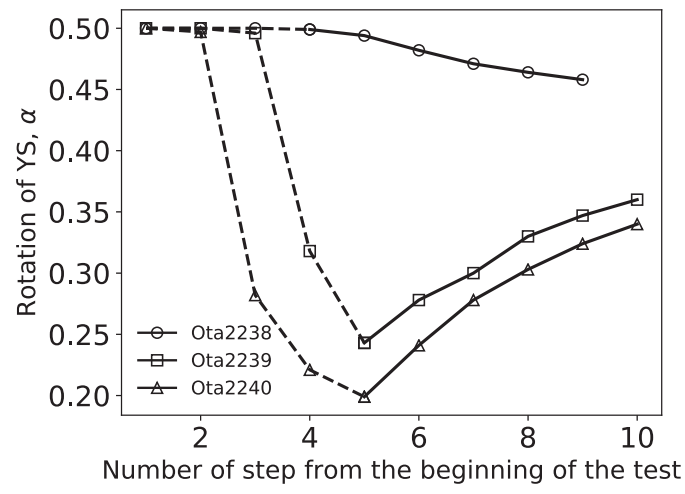

(a) Anisotropy

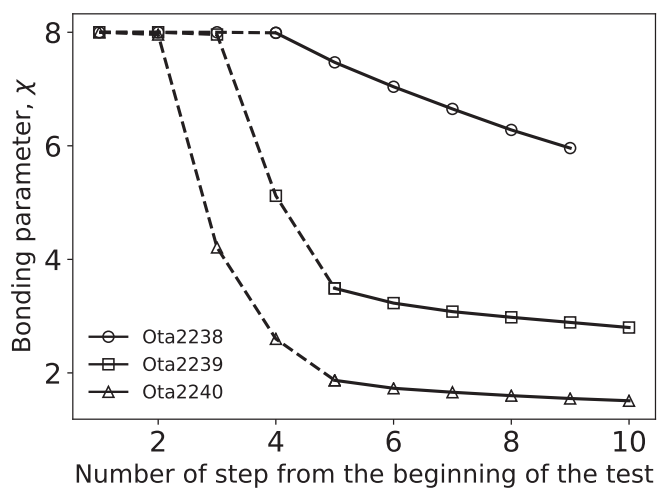

(b) Structure

Fig. 4. Undrained shearing tests on Otaniemi clay [5] and numerical simulations with Creep-SCLAY1S. Stage 1 (Dashed lines): anisotropic consolidation; stage 2 (Solid lines): undrained shearing.

\section{Conclusions}

In this work, the important role of the evolution of anisotropy and destructuration of soft structured clays is demonstrated based on simulations on undrained triaxial shearing tests of Gothenburg and Otaniemi clays. Simulations of Gothenburg clay by hierarchically increasing the model complexity demonstrate that features such as anisotropy and destructuration are important to account for in modelling the clay response over the whole effective stress range, The simulations of Otaniemi clay, on the other hand highlight the need to account for the changes in the state of the soil during the initial consolidation.

The financial support by the Swedish Research Council FORMAS (contract number 2016-00834) and by Swedish Transportation authority under the umbrella of BIG (Brach Collaboration in Ground, contract number TRV 2016/106280) is greatly acknowledged.

\section{References}

[1] S. Leroueil, P.R. Vaughan, Geotechnique 3, 467 (1990)

[2] M. Koskinen, M. Karstunen, S. Wheeler, Modelling destructuration and anisotropy of a natural clay, in 5th European Conf. Numerical Methods in Geotechnical Engineering, edited by P. de L'ENPC/LCPC (Paris, 2002), pp. 11-20

[3] N. Sivasithamparam, M. Karstunen, P. Bonnier, Computers and Geotechnics 69, 46 (2015)

[4] J. Gras, N. Sivasithamparam, M. Karstunen, J. Dijkstra, Computers and Geotechnics 90, 164 (2017)

[5] M. Koskinen, Ph.D. thesis, Aalto University (2014)

[6] S. Wheeler, A. Naatanen, M. Karstunen, M. Lojander, Canadian Geotechnical Journal 40, 403 (2003)

[7] A. Gens, R. Nova, Conceptual bases for a constitutive model for bonded soils and weak rocks, in In- 
ternational Symp. on Hard Soils-Soft Rocks (Athens, 1993), pp. 485-494

[8] Y.F. Dafalias, Mechanics Research Communications 13, 341 (1986)

[9] G. Grimstad, S.A. Degago, S. Nordal, M. Karstunen, Acta Geotechnica 6, 69 (2010)

[10] M. Karstunen, H. Krenn, S. Wheeler, M. Koskinen, R. Zentar, International Journal of Geomechanics 9, 153 (2009) 